\title{
Applications of energy dependent backscatter yield variations at low voltage.
}

\author{
Markus Boese ${ }^{1}$, Kerstin Sempf ${ }^{2}$, Fang Zhou ${ }^{1}$ and Alexander Thesen ${ }^{1}$ \\ 1. Zeiss Microscopy, SEM Department, Oberkochen, Germany. \\ 2. Fraunhofer- IKTS, Ceramography and Phase Analysis, Dresden, Germany
}

For imaging of nano-materials and composites it is often necessary to detect smallest compositional differences in these materials. Very often the nanostructure needs to be distinguishable from matrix or substrates in order to image it. The main advantage of material analysis at low Voltages is due to the reduced interaction volume of the primary beam. Benefit is here a higher resolution and the ability to gain surface sensitivity for better imaging conditions.

It is known, that under certain low Voltage conditions a contrast reversal occurs, showing high Z materials with dark contrast and light materials with bright contrast in the BSE image. In this study we investigate, how the change of the backscatter coefficient with changing primary energy can be utilized for gaining information in SEM backscattered imaging. In particular, if this can be used to gain material contrast for low voltage backscattered imaging compared to imaging conditions above 1-2 kV.

The primary energy dependency of the backscatter coefficient was described by Cazaux [1] using the empirical expression:

$$
\eta=a\left(1 \pm \mathrm{e}^{-b \mathrm{Eo}}\right)
$$

The sign is + for low Z and - for the high Z elements. The parameters " $a$ " and " $b$ " were fitted to experimental values and a resulting characteristic is given for a range of different elements $\mathrm{Z}$ range from 4 to 80 . The different slope for different elements is resulting from the different $b$ values, whereas the a value is corresponding to the asymptotic value for higher Eo $(>2-4 \mathrm{kV})[1]$.

Figure 1 shows the energy dependency of the yield, plotted for high $\mathrm{Z}$ and low Z elements. In general backscattering yield becomes lower for heavier elements going to low voltage and higher for lighter elements. The shape of the curve depends strongly on " $b$ ". The discontinuous change of this parameter (as shown in Fig.2) enables us to produce some extra contrast for certain element combinations under low voltage imaging conditions. We can expect a raise in contrast up to 40\% for imaging Ag and $\mathrm{Zn}$ at $300 \mathrm{~V}$ (Fig. 1) compared to $10 \%$ contrast for primary energies higher than $2 \mathrm{kV}$. (Note at high energy the backscatter yield is solely dependent on the " $a$ ” parameter)

For low Z the parameter " $b$ ” changes as well for different elements. But the contrast (difference between the $\mathrm{Al}$ and $\mathrm{O}$ yield in Fig. 1) is not changing as much. Here the advantage of low voltage applications is the higher signal itself due to the higher yields at low Voltage.

Experiments are carried out in a ZEISS Ultra 55 SEM equipped with an EsB detector capable of BSE detection in a sub $1 \mathrm{kV}$ regime. The GEMINI lens design enables the detection of backscattered electrons passing through the objective lens and then hit the EsB Detector positioned above the Inlens SE detector. A grid repels secondary electrons and can be used for energy filtered SEM Imaging [2]. Fig. 3 illustrates the low voltage imaging capabilities of this system. 
Another example is given by a ceramic composite sample consisting of high $\mathrm{Z}$ and low $\mathrm{Z}$ phases. As shown in Fig 4 the low voltage BSE image is suitable to differentiate 5 different phases due to the leveling of the individual backscattering signal for high and low $\mathrm{Z}$.

Further applications for the contrast enhancement at low voltage could be done on material systems like InGaAs, and $\mathrm{Pb}$ free solder containing different phases of $\mathrm{Cu}, \mathrm{Ag}$ and $\mathrm{Sn}$.

References:

[1] J. Cazaux, J. Appl. Phys 112 (2012), p. 84905.

[2] H. Jaksch, EMC 2008 proceedings (2008), 1, 555

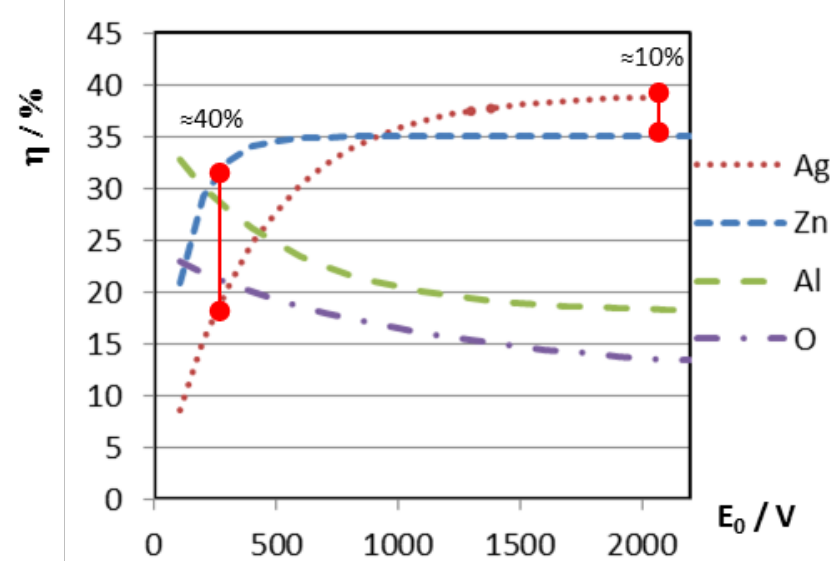

Figure 1. Energy dependency of backscatter yield $\eta$ for high and low $\mathrm{Z}$ materials. The different slope of the curves can be utilized to gain contrast at low voltage imaging conditions

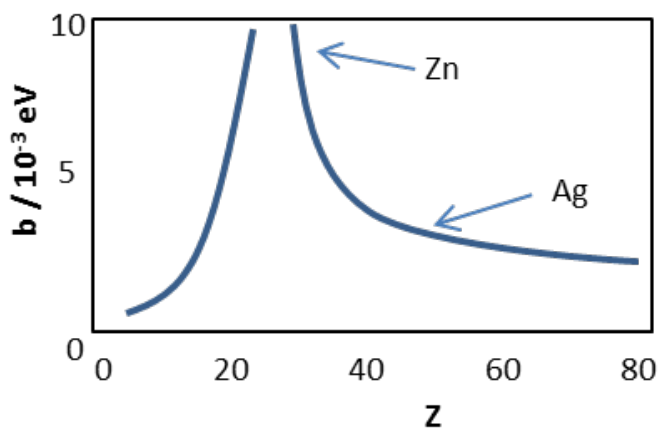

Figure 2. Empirical parameter " $b$ " plotted for different elements. The strong variation here causes the different slopes in Fig. 1

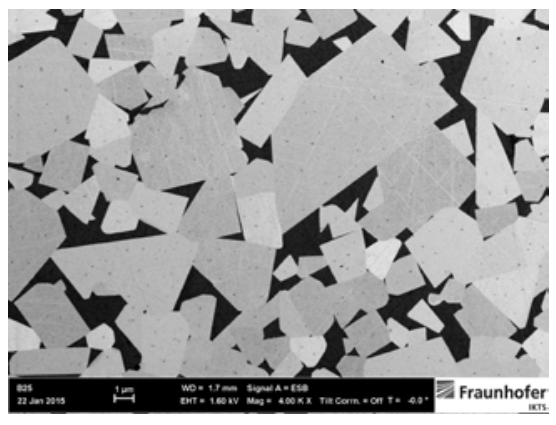

Figure 3. For different primary energies (left image Eo=1600V and right image 500V) a contrast inversion is observed. Sample: tungsten carbide with cobalt sintering agent (in between the grains). The slight contrast variation within the tungsten carbide is due to channeling.

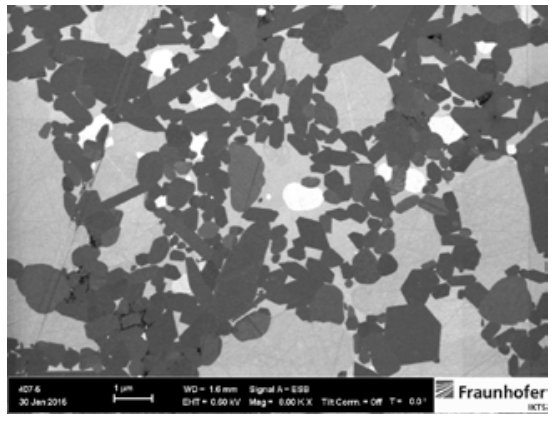

Figure 4. 5 different phases are distinguishable under low voltage conditions. Phases are: $\mathrm{SiC}, \mathrm{Si}_{3} \mathrm{~N}_{4}$, $\mathrm{Y}$-Al-Si-oxinitride, $\mathrm{MoSi}_{2}, \mathrm{Mo}_{5} \mathrm{Si}_{2}$ 